\title{
The Rheology and Microstructure of Carbon Nanotube (CNT) Suspensions
}

\author{
Anson $\mathrm{Ma}^{*}$, Francisco Chinesta ${ }^{\dagger, *}$ and Malcolm Mackley ${ }^{*}$ \\ ${ }^{*}$ Department of Chemical Engineering, University of Cambridge, \\ Pembroke Street, CB2 3RA, United Kingdom \\ ${ }^{\dagger}$ Laboratoire de Mécanique des Systèmes et des Procédés \\ UMR 8106 CNRS-ENSAM-ESEM \\ 151 Boulevard de l'Hôpital, F-75013 Paris, France
}

\begin{abstract}
This conference paper is concerned with the rheology and microstructure of Carbon Nanotube suspensions. Carbon Nanotubes (CNTs) are cylinders of rolled graphene sheets of high aspect ratio and they belong to a relatively new class of fibrous material that can potentially be used for high-performance nano-composites and nano-devices. For these applications, understanding the rheology and microstructure of CNT suspensions is of key importance as this would allow for processing CNTs into macroscopically usable form with controlled properties. This paper reports the rheology and optical microstructure of surface-treated and untreated CNT suspensions. In terms of rheology, both types of CNT suspensions exhibited a shear-thinning characteristic in steady simple shear flows and their rheological responses were subsequently modelled using a FokkerPlanck (FP) based orientation model that incorporates aggregation effect in the case of untreated CNT suspension. In terms of unusual mesostructures, it was observed that by applying appropriate flow conditions to an untreated CNT suspension, a novel type of mesoscopic filament structure could be formed, where the CNT filaments aligned perpendicular to the flow direction and in the vorticity direction. The formation of these anisotropic structures has similarities to the hand-spinning of cotton into fibres and these flow-induced structures might have strategic benefits for some potential applications of CNT.
\end{abstract}

Keywords: Carbon Nanotube suspension; Processing; Rheo-optics; Shear-induced assembly PACS: 61.46.Fg; 61.48.+c; 81.16.ąVc; 81.16.Fg

\section{INTRODUCTION}

Carbon Nanotubes (CNTs) are rolled cylinders of graphene sheets having high aspect ratio, low density, high mechanical strength and high thermal conductivity [1] [2]. Because of these intrinsic properties, they have been researched in a number of application areas such as high-performance nanocomposites and nanodevices [3] [4]. For most of these applications, understanding the rheology and microstructure of CNT suspensions is important in relation to processing this type of nanomaterials into some macroscopically usable forms with predictable properties. The objectives of this paper are to highlight several key experimental findings, both in terms of rheological and optical studies of CNT suspensions, and secondly, to discuss briefly the basis on which CNT suspensions can be modelled using a Fokker-Planck (FP) based diffusion-advection model. Finally, flow-induced formation of some unusual CNT mesostructures is also described. 


\section{CARBON NANOTUBE SUSPENSIONS}

At present, enhancing the bulk conductivity of a polymer film is identified as one of the most promising potential applications for CNTs [5] [6] and for such an application, it is thought desirable to have a well-dispersed system of CNT. However, this can be a laborious and challenging task because in general, a mixing time of several hours is required in order to establish a stable rheology for CNT suspensions, and even then dispersion at the nano-scale is not guaranteed [7]. In this regard, introducing functional groups onto the surface of CNTs, also known as functionalization, has proven to be one possible way of enhancing the dispersion of CNT in a processing matrix [8]. The actual chemistry involved in surface-treating CNTs may differ, but functionalization generally works on the principle of increasing steric hindrance or introducing electrostatic repulsion between CNTs, thereby preventing effective $\pi-\pi$ stacking and subsequent aggregation of CNTs (see for example [9]). Figure 1 shows typical optical micrographs of surface-treated and untreated CNTs suspended in epoxy resin ${ }^{1}[10]$. It is clear from the micrographs that the untreated CNT suspension exhibited an optical microstructure (Fig. 1(a)), whilst by contrast, there is no discernable microstructure for the treated CNT suspension (Fig. 1(b)), indicating that the mixture was well-dispersed at the micron-level.

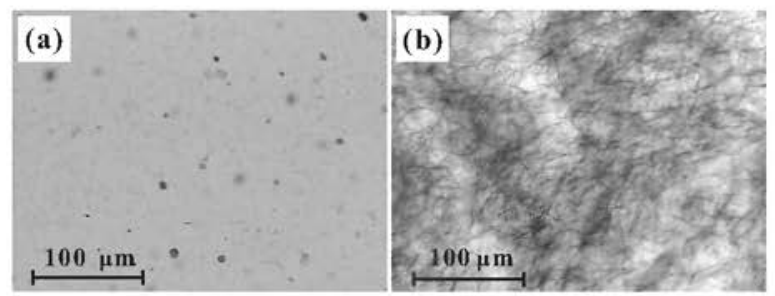

FIGURE 1. Optical micrographs of $0.3 \%$ (a) surface-treated and (b) untreated CNT suspensions [10].

\section{RHEOLOGY}

The rheology of both treated and untreated CNTs suspended in epoxy resin were studied and modelled for simple rheometric flows. It was observed experimentally that both types of suspensions exhibited a shear-thinning characteristic when subject to steady simple shear and the apparent viscosity $\left(\eta_{a}\right)$ of the suspension decreased asymptotically to the matrix viscosity as shear rate increased (Fig. 2). Given the same weight concentration of CNTs, the untreated CNT suspension exhibited a significantly larger viscosity enhancement effect at low shear rates compared with the treated CNT suspension, as also shown in Fig. 2.

\footnotetext{
${ }^{1}$ The surface-treated CNTs were single-walled and were supplied by Nanocomposites Inc., whereas the untreated CNTs were multi-walled and were provided by the Department of Materials Science and Metallurgy, University of Cambridge. The epoxy resin used was Araldite LY556 from Huntsman Inc.
} 


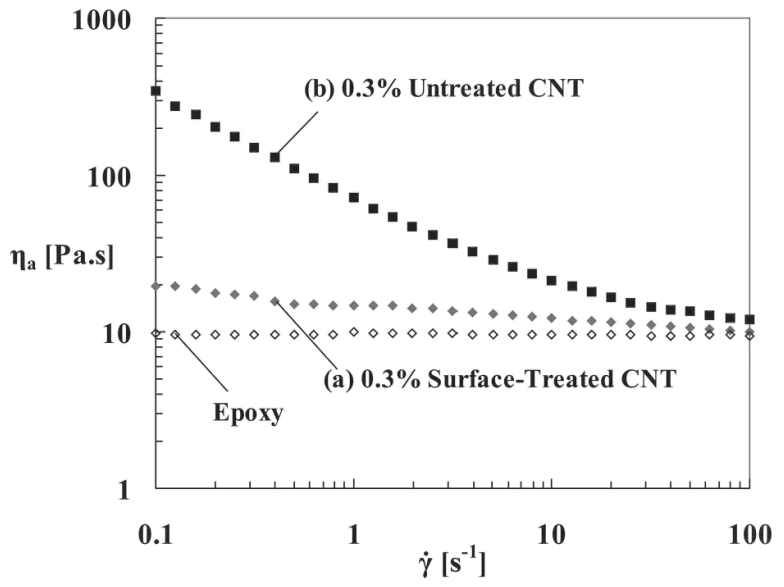

FIGURE 2. Apparent viscosity $\left(\eta_{a}\right)$ as a function of shear rate $(\dot{\gamma})$ for (a) surface-treated and (b) untreated CNT suspensions [10].

For treated CNT suspensions, the observed shear-thinning characteristic can be modelled using a simple orientation model that in the past has been used for short-fibre suspensions [11]. The model basically assumes that CNTs behave essentially as rigid rods and that they can orient and align in a simple shear flow. There are, however, also randomizing events due to Brownian motion and tube interaction (for semi-dilute or concentrated suspensions), which can be represented using a rotary diffusion coefficient $\left(D_{r}\right)$ [12] and the time evolution of CNT orientation distribution can be described using a Fokker-Planck based diffusion-advection equation [13]. $D_{r}$ and an adjustable parameter $N_{p}$ that depends on the concentration and aspect ratio are the key parameters in the model and such model can successfully describe the shear-thinning characteristics of treated CNT suspensions as shown in Fig. 3. Besides simple shear flows, the model was also capable of describing some kinetic responses of CNT suspensions in smallamplitude oscillatory shear flows, thereby supporting the basis for the model itself [10].

However, in the case of untreated CNT suspensions where aggregates of CNTs were optically observed, the simple orientation model failed to describe the shear-thinning characteristic observed experimentally, and therefore a more sophisticated model is needed. An Aggregation/Orientation (AO) model has been proposed in this context by considering extra contribution from CNT aggregates [14]. The model assumes that in an aggregating suspension of CNTs, CNTs can exist in different entanglement states and they can have different mobility to orient in a simple shear flow. The Fokker-Planck equation was then modified to accommodate for a hierarchy of microstructures. In this model, transformation between microstructures is possible depending on the shear rate applied to the system, and thus matching the phenomena of CNT aggregation and disaggregation as observed experimentally [15] [16]. 


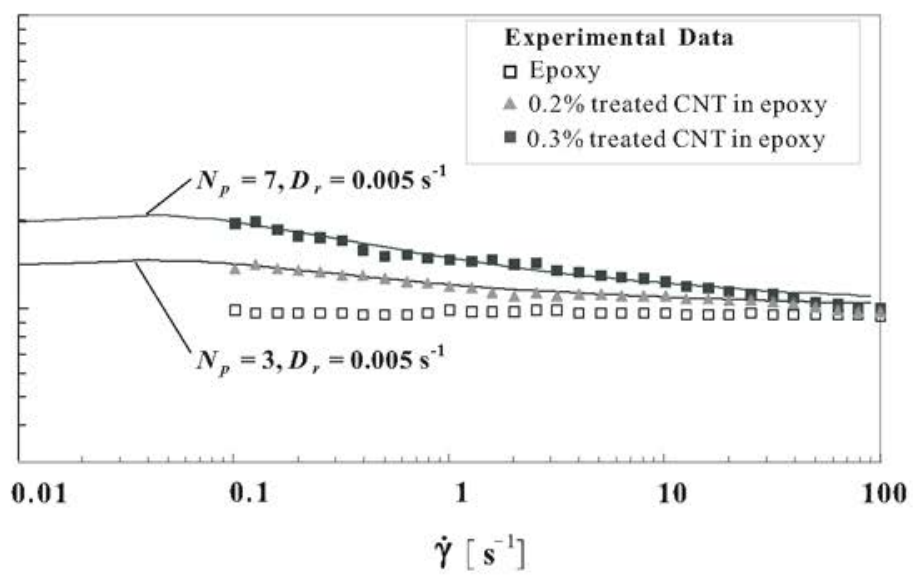

FIGURE 3. Simple orientation model fitting of $0.2 \%$ and $0.3 \%$ treated CNT suspensions. $D_{r}$ and $N_{p}$ are the key fitting parameters in the model. $\left(\eta_{a}=\right.$ apparent viscosity and $\dot{\gamma}=$ shear rate.) [10]

\section{UNUSUAL FLOW MICROSTRUCTURE}

The flow microstructure of untreated CNT suspensions was followed using the Cambridge Shear System (Linkam Instruments Inc.), which essentially consists of a shear cell coupled with an optical microscope. Although shear can induce alignment of CNTs, it was also reported that low shear could induce aggregation of CNTs [15] [16]. In most cases, a homogeneous dispersion of CNT is desired, however there are other situations where a highly heterogeneous structure can be of strategic benefit. It was discovered that some unusual filament mesostructures could be formed by applying appropriate flow conditions to an untreated CNT suspension [17]. Figure 4 shows the time evolution of optical texture as low shear was applied to some untreated CNTs $(0.03 \%)$ suspended in a low-viscosity epoxy matrix ${ }^{2}$. Initially, there were only isotropic aggregates of CNTs (Fig. 4(a)), but as they rotated within a simple shear flow, they drew in nearby CNT aggregates to form long Mesoscopic Helical Filaments (MHFs) that aligned perpendicular to the flow and in the vorticity direction. The way in which CNT aggregates are helically wound to form this kind of filament structure is intriguing and not yet fully understood. In terms of applications, although the advantage of having a MHF structure of CNTs has not yet been explored, it is expected that the anisotropic nature of these mesostructures will produce preferential conductivity along the length of the filaments and thereby providing unusual anisotropic electrical properties to the system as a whole.

\footnotetext{
${ }^{2}$ The low-viscosity epoxy has a viscosity of 0.4 Pa.s and was supplied by Epoxies Inc. (UV 60-7155).
} 

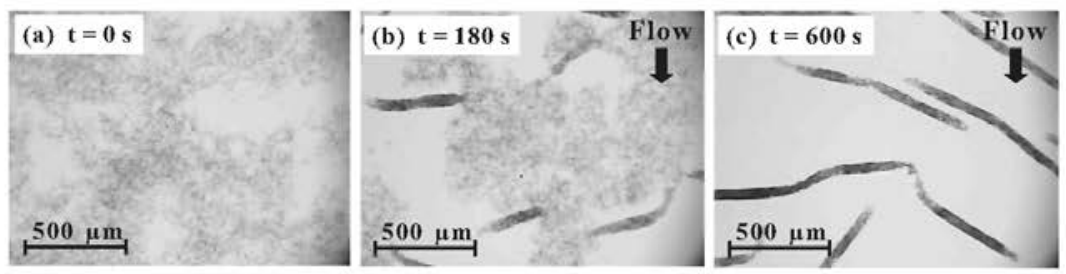

FIGURE 4. Time evolution of optical texture as low shear $\left(\dot{\gamma}=0.5 s^{-1}\right)$ was applied to $0.03 \%$ untreated CNTs suspended in a low-viscosity epoxy matrix (gap size $=130 \mu \mathrm{m}$ ) [17].

\section{CONCLUSIONS}

In conclusion, this paper reported recent findings on the rheo-optical studies of Carbon Nanotube (CNT) suspensions which are of general relevance to the processing of CNTs. It was found that for surface-treated CNT suspensions that exhibit no discernable optical microstructure, CNTs can essentially be modelled as rigid fibres that can orient in a shear flow. The rheology of flowing CNTs can be successfully described using a simple orientation model and essential information on the orientation distribution of CNTs can be obtained from a Fokker-Planck (FP) description. The FP based orientation model was capable of predicting both steady and transient responses of treated CNT suspensions, but failed in the case of untreated CNT suspensions where CNT aggregates were optically observed. This has led to the development of a more appropriate Aggregation/ Orientation (AO) model, in which a hierarchy of CNT aggregate structures was considered. Finally, in terms of optical microstructure, although low shear can induce aggregation and should be avoided in cases where a uniformly dispersed system of CNTs is desired, it can also be used strategically to produce some unusual Mesoscopic Helical Filament (MHF) structures that are highly anisotropic.

\section{ACKNOWLEDGMENTS}

We would like to thank Nanocomposites Inc. and Prof. A. H. Windle from the Department of Materials Science and Metallurgy at the University of Cambridge for providing the base Carbon Nanotubes. Anson Ma would also like to to acknowledge the Croucher Foundation Scholarship (Hong Kong) and the Overseas Research Students Awards Scheme (ORSAS) (United Kingdom) for providing financial support. 


\section{REFERENCES}

1. S. Iijima, "Helical microtubules of graphitic carbon," Nature 354, 56-58 (1991).

2. M. S. Dresselhaus and H. Dai, "Carbon Nanotubes: continued innovations and challenges," MRS Bulletin 29, 237-243 (2004).

3. P. Calvert, "Nanotube composites: A recipe for strength," Nature 399, 210-211 (1999).

4. W. A. de Heer, A. Chatelain, and D. Ugarte, "A Carbon Nanotube field-emission electron source," Science 270, 1179-1180 (1995).

5. J. Sandler, M. S. P. Shaffer, T. Prasse, W. Bauhofer, K. Schulte, and A. H. Windle, "Development of a dispersion process for Carbon Nanotubes in an epoxy matrix and the resulting electrical properties," Polymer 40, 5967-5971 (1999).

6. M. S. P. Shaffer, X. Fan, and A. H. Windle, "Dispersion and packing of Carbon Nanotubes," Carbon 36, 1603-1612 (1998).

7. Y. Y. Huang, S. V. Ahir, and E. M. Terentjev, "Dispersion rheology of Carbon Nanotubes in a polymer matrix," Phys. Rev. B 73, 125422-1-9 (2006).

8. S. Banerjee, M. G. C. Kahn, and S. S. Wong, "Rational chemical strategies for Carbon Nanotube functionalization," Chem. Eur. J. 9, 1898-1908 (2003).

9. C. A. Dyke and J. M. Tour, "Unbundled and highly functionalized Carbon Nanotubes from aqueous reactions," Nano Letters 3(9), 1215-1218 (2003).

10. A. Ma, F. Chinesta, and M. R. Mackley, "Rheological modelling of suspensions involving surfacetreated Carbon Nanotubes," submitted to J. Rheol..

11. C. J. S. Petrie, "The rheology of fibre suspensions," J. Non-Newtonian Fluid Mech. 87, 369-402 (1999).

12. F. Folgar and C. L. Tucker III, "Orientation behaviour of fibers in concentrated suspensions," J. Reinf. Plast. Compos. 3, 98-119 (1984).

13. E. J. Hinch and L. G. Leal, "The effect of Brownian motion on the rheological properties of a suspension of non-spherical particles," J. Fluid Mech. 52, 683-712 (1972).

14. A. Ma, F. Chinesta, and M. R. Mackley, "Rheological modelling of suspensions involving untreated Carbon Nanotubes in shear flow," submitted to J. Rheol..

15. S. S. Rahatekar, K. K. K. Koziol, S. A. Butler, J. A. Elliott, M. S. P. Shaffer, M. R. Mackley, and A. H. Windle, "Optical microstructure and viscosity enhancement for an epoxy resin matrix containing multi-wall Carbon Nanotubes," J. Rheol. 50, 599-610 (2006).

16. S. Lin-Gibson, J. A. Pathak, E. A. Grulke, H. Wang, and E. K. Hobbie, " Elastic flow instability in nanotube suspensions," Phys. Rev. Lett. 92, 048302-1 - 4 (2004).

17. A. Ma, M. R. Mackley, and S. S. Rahatekar, "Experimental observation on the flow-induced assembly of Carbon Nanotube suspensions to form Mesoscopic Helical Filaments," submitted to Rheol. Acta. 